\title{
Teaching and research in astronomy using small aperture optical telescopes
}

\author{
Shiva Pandey $\dagger$ \\ School of Studies in Physics, Pt. Ravishankar Shukla University, Raipur, India \\ email: skp@iucaa.ernet.in
}

\begin{abstract}
Small aperture ( $<1 \mathrm{~m}$, typically $20-50 \mathrm{~cm}$ ) optical telescopes with adequate backend instrumentation (e.g. photometer, CCD camera and CCD spectrograph) can be used for spreading the joy and excitement of observational astronomy among postgraduate and research students in colleges and universities. On the basis of our experience over a decade of observing with small optical telescopes it has been amply demonstrated that such a facility, which any university can hope to procure and maintain, can be effectively used for teaching and research. The Physics Department of Pt. Ravishankar Shukla University at Raipur, India offers Astronomy \& Astrophysics as one of the specializations of its MSc program in Physics. A set of observational exercises has been incorporated with a view to provide training in observations, analysis and interpretation of astronomical data. Observing facilities available in the department include 8"14" aperture telescopes equipped with a photometer, CCD camera and a CCD spectrograph. A facility of this kind is ideally suited for continuous monitoring of a variety of variable stars, and thus can provide valuable data for understanding the physics of stellar variability. This is especially true for a class of variable stars known as chromospherically active stars. The stars belonging to this class have variable light curves that change from year to year in a rather strange way. A large fraction of these active stars are bright; hence the importance of small aperture telescopes for collecting much-needed photometric data. For over a decade the research activity using the 14" optical telescope has focused on photometric monitoring of well known and suspected active stars. These data, together with X-ray and radio data from archives as well as spectroscopic data obtained at Indian observatories, has led to the identification of new chromosperically active stars. This paper is aimed at sharing our experiences with the colleagues from the developing world on the usage of small optical telescopes for teaching and research with the objective of spreading the joy of astronomy among young students.
\end{abstract}

Keywords. Astronomy: teaching \& research, stars: photometry, stars: active, stars: variable

\section{Introduction}

Undoubtedly, optical telescopes are amongst the most powerful tools in astronomy for probing the Universe we live in. The quest to explore the mysteries of the cosmos has led to the development of optical telescopes of bigger and bigger aperture. Optical telescopes of all sizes are useful for collecting data on a variety of objects in the Universe, and all have contributed to providing a better understanding of the Universe. It is not important what one has or can have; what is more important is what one does using what capability one has. For the purpose of this article, optical telescopes with aperture less than a metre are termed small telescopes. Development of technology for back-end instrumentation has yielded low-cost stellar photometers, CCD cameras and spectrographs. Together with the increased sophistication in telescope technology and data acquisition and reduction software packages, this has led to an enormous improvement in both the accuracy and the efficiency of observations using small optical telescopes. These features of modern small

$\dagger$ Visiting Associate, IUCAA, Pune, India. 
optical telescopes are of great importance when one talks about introducing astronomy as a subject at various levels in schools, colleges or universities. Small optical telescopes equipped with suitable back-end instrumentation are capable of performing several tasks to enhance the teaching of astronomy. Small telescopes can also be employed for initiating research activities on suitably and carefully chosen projects on a variety of variable stars, leading to publication in reputed journals as well. In this article I will describe the activities that we have been carrying out with small optical telescopes, highlighting some research activities which have been carried out quite successfully. This has so far paid rich dividends in terms of creating manpower in astronomy. This, together with the facilities available at IUCAA and its Reference Centre in the department, has provided a big boost for promoting teaching and research in astronomy and astrophysics in our University.

\section{Growth of astronomy and astrophysics in the Physics Department}

Astronomy \& Astrophysics (A\&A) has been one of the major areas of research in the Physics Department since its establishment in 1972, but teaching in A\&A started a few years later at the initiative of Professor R K Thakur, the then Head of the Physics Department, and gradually got strengthened with the addition of small optical telescopes (6" from Carl Zeiss in 1978, and a computerized 14" Celestron telescope in 1988) and stellar photometers (SSP3 and SSP5 from OPTEC). Completion of an observatory dome for housing the $14^{\prime \prime}$ telescope in 1991-92 sparked the beginning of observational astronomy in the department, and a new set of activities were initiated. These included a new astronomy syllabus with observational projects to supplement the theory course for the postgraduate students. The establishment of the Inter-University Centre For Astronomy and Astrophysics (IUCAA) at Pune, India in 1988 gave a big boost to our efforts. In order to find ways in which to utilize the telescopes maximally, we searched for suitable research projects for small telescopes. After a brief literature survey on variable stars, mostly done by Mr Padmakar Parihar, we selected a small sample of bright and prominent RS CVn-type active stars, and began photometric monitoring of these stars in 1994 . Research projects sponsored by Indian funding agencies, such as DST, CSIR, etc., added a new dimension to our research programme by way of providing financial support for the research scholars and for maintenance of the observatory equipment. During the period 1994-2002 the facility was used for collecting good quality photometric data on several active stars. This was incorporated as a part of the thesis work of Padmakar (2000). The observational astronomy programme in the department received a severe blow when the observatory building collapsed in 2002. To make things worse, the 6" Carl-Zeiss telescope was stolen during December 2003, which brought about the collapse of the entire observational programme of the department. Fortunately, with the support from IUCAA, somehow we managed to continue our teaching programme in astronomy for the students using the small telescopes at IUCAA in Pune. A grant received from DST-FIST in 2005 helped the department to procure new telescopes and instrumentation. Since then the training programme in observational astronomy has resumed in the department.

The new observing facility in the Physics Department includes:

(i) two small telescopes, CGE800 and CGE1400, from Celestron with GPS-assisted pointing and other accessories;

(ii) a stellar photometer SSP3A from Optec;

(iii) a CCD camera ST-7XME along with spectrograph from SBIG;

(iv) software and other accessories. 
The facilities are shown in Figs. 1 and 2. The department also has an adequate computing facility for data reduction and analysis, as well as a small library and internet connectivity using a VSAT facility sponsored by the University Grants Commission.

The School of Studies in Physics currently offers a two-year MSc course in physics with astronomy and astrophysics (A\&A) as one of the specializations. The MSc course is split into four semesters and comprises both core and elective subjects in different areas of physics. A\&A is taught as one of elective courses spread over two semesters (III \& IV). The course includes topics on stellar structure and evolution, the Milky Way and other galaxies, active galaxies, general relativity and elementary cosmology. The theory course is supplemented with a project which can be either observational or theoretical. The astronomy course is quite popular in the department. Each year, about 10-12 students opt for A\&A as one of their elective subjects.

It is worth mentioning here that Pt. Ravishankar Shukla University is among the few Universities in India (and the only one in the new state of Chattisgarh) where teaching and research in A\&A is conducted in the Physics Department.

The mission of the Inter-University Centre for Astronomy and Astrophysics (IUCAA) is to initiate and promote teaching and research in A\&A in the Indian Universities. It provides support to students, research scholars and faculty, allowing them to make use of the facilities at the Centre. Since its establishment, IUCAA has played a significant role in the sustaining the research interests of the faculty members of the department. In 1999 IUCAA created its Reference Centre in the Physics Department for the promotion of teaching and research in $\mathrm{A} \& \mathrm{~A}$ in this region as a mark of recognition for the contribution that Department of Physics has made in the field of A\&A. IUCAA provides support to short-term visitors, a small library and internet dial-up facility. The IUCAA support has become a life-line for strengthening the teaching and research activities in astronomy at the University.

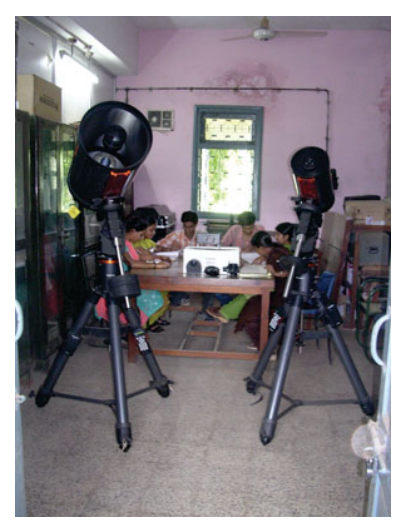

Fig 1: Small optical telescopes: $8^{\prime \prime} \& 14^{\prime \prime}$.

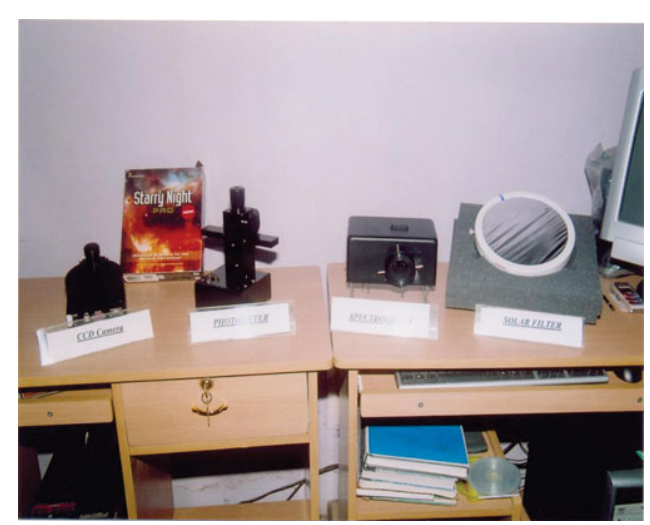

Fig 2: Back-end instruments.

\section{Activities in astronomy using small telescopes}

Small optical telescopes are very useful for teaching astronomy in schools, colleges and universities. The availability of low-cost instrumentation (e.g. stellar photometers, CCD cameras and stellar spectrographs) has enhanced the capability of small telescopes in the present era of ever increasing sizes of large optical telescopes. In addition to selecting a set of observational projects to complement theoretical courses at graduate and 
post-graduate level, one can plan and implement research projects for which small telescopes are ideally suited (see for example Percy (1986) and Paczynski (2006)). The selection of an observing program depends on several factors such as (a) available equipment, (b) observing site, (c) time-scale of required observations, (d) experience, etc. One may seek help and advice from professional astronomers, consult the AAVSO, newsletters, etc. It turns out that photometry of well known short-period variable stars is good choice to begin with.

\subsection{Observational training projects}

Final-year students of the Department who offer Astronomy as the specialization for their MSc course carry out observational projects. For the postgraduate students, the required time-scale becomes one of the most important factors when selecting objects for them to observe. The objects for the project are chosen such that a student can carry out observations within a month, taking in to account all overheads. The observational projects include: (i) atmospheric extinction measurements, (ii) calibration of the photometer and determination of transformation coefficients, (iii) determining the H-R Diagram of nearby bright stars and star clusters like Pleiades and Hyades, and (iv) tracing light curves of various types of bright variable stars, such as Cepheids, Delta Scuti stars, eclipsing binaries like Algol and $\lambda$-tau and bright active (e.g. RS CVn) stars. During the course of the project work students are trained in observing techniques, data acquisition, reduction and analysis as well as in interpretation of the results.

\subsection{Public outreach}

Astronomy has proved to be a very effective platform for communicating science to young minds and tuning their excitement to science because of its unique quality of mass appeal. Celestial objects in the night sky have always been subject of awe and wonder to every one! This popularity of astronomy, the mother of all sciences, can be maximally utilized in attracting young students into the stream of pure science subjects. With this in mind the Physics Department started a sky gazing programme when it procured the 6" optical telescope in 1978. The programme continues to this day during the observing season and whenever special events become observable in the sky.

\subsection{Research}

Small telescopes equipped with adequate instrumentation are ideally suited for carrying out photometric observations of variable stars, and thus can provide valuable data for understanding the physics of variability. This is especially true for a class of variable stars known as chromospherically active stars, or RS Canum Venaticorum stars, after the prototype of this class identified by Hall and his group during 1972-1976. The stars belonging to this class have variable light curves, and the most puzzling feature is that their light curves change year after year in a rather strange way. The light variation is attributed to rotational modulation of dark spots, similar to sunspots, on the stellar surface, but a clear understanding of the evolution and dynamics of the dark spots remains one of the most puzzling issues in stellar physics. This bears far reaching consequences as regards the origin of the magnetic field and related dynamo problem in these stars. Continuous photometric monitoring of these active stars is therefore required to examine short-term as well long-term variations in their light curves. A large fraction of these active stars are bright enough to be monitored with small telescopes for collecting the much-needed photometric data.

With these objectives in mind we used our computer-controlled 14" Schmidt-Cassegrain Celestron to obtaining $B V R$ photometry of several prominent RS CVn binaries. These 
observations were used in the theses of Padmakar Parihar and Sudhnashu Barway. These stars are found to display appreciable changes in amplitude, pulse shape, phase of minimum light as well as mean light level within a couple of rotation periods. Fig. 3. shows sample light curves of the short-term variation in the RS CVn binary V711 Tau. This is the nearest $(29 \mathrm{pc})$ and brightest $\left(V_{\max }=5.74\right)$ chromospherically active binary star with a short period (2.84 days) having a K1 IV primary and G5 V secondary.

In addition to regular monitoring of prominent RS CVn stars, several X-ray binaries which are suspected to be chromospherically active stars were monitored photometrically using the 16" Meade telescope at IUCAA during the period 2000-2001. Analyses of the optical photometric data in conjunction with spectroscopic, X-ray and radio continuum data of HD 61396 strongly suggested its identification as a new RS CVn-type binary (Padmakar et al., 2000a).

Likewise, Sudhnashu Barway as a part of his thesis work carried out photometric observations of five suspected variable stars selected on the basis of their intense X-ray emission as revealed from the space observatory ROSAT. Barway observed these stars during 2000-2001 using a 16" Meade telescope equipped with an SSP-3A photometer. Preliminary results indicate that four of these stars belong to the class of chromospherically active stars (Barway, 2005). Fig. 4 shows sample light curves of one of these stars, HD 39286.

These discoveries of new chromospherically active stars, apart from enriching the existing sample of active stars, are very important for understanding the underlying physical parameters like rotation, age, metallicity, etc. which are involved in generating and sustaining strong chromospheric and coronal activity in stars.

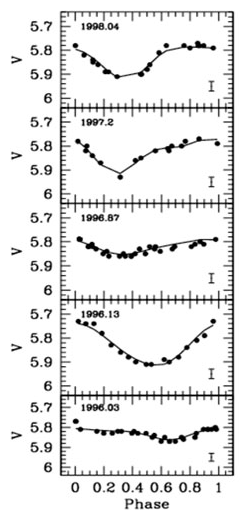

Fig 3: Short-term light variation in RS CVn star V711 Tau.
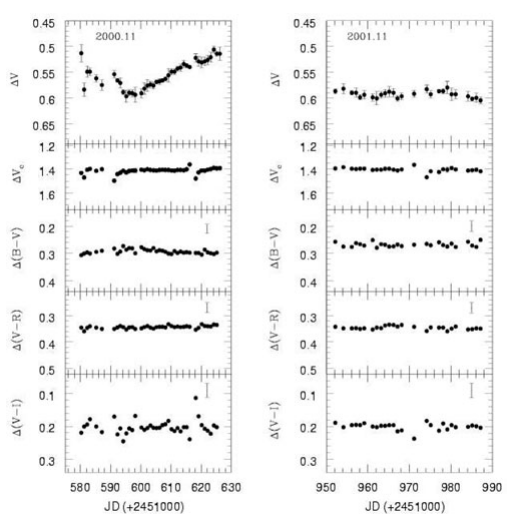

Fig 4: Light curves of HD39286 for two epochs.

\section{Assessing the impact of small telescopes}

If one asks whether our efforts to promote the usage of small telescopes have made any impact in the astronomy scene in India, the answer is a big 'yes' based on the feedback and compliments the Physics Department has received from astronomer colleagues in the country, specially in terms of generating manpower in astronomy. Given the constraints, and limitations of the working environments under which the department functions, the recognition earned by the department is indeed a big moral boost and encouraging for the group involved in the observational astronomy activities in the Physics Department.

Research activities using small telescopes at the department and at IUCAA have led to the following publications in journals during the period 1996-2005. 
1. BVR Photometry of the three RS CVn Binary Stars: V711 tau, UX Ari and IM Peg: Padmakar and Pandey (1996a).

2. A study of long term photometric variation in the RS CVn Star V711 Tau: Padmakar and Pandey (1996b).

3. Stellar activity in the RS CVn binary star UX Arietis: Padmakar and Pandey (1999a). 4. New BVR photometry of six prominent RS CVn binary stars: Padmakar and Pandey (1999b).

5. Optical, X-ray and radio observations of HD 61396; a probable new RS CVn type binary: Padmakar et al.(2000a).

6. Study of sky conditions at Raipur and calibration of photometer-telescope system: Padmakar, Barway and Pandey (2000b).

7. HD 52452: New BVRI photometry : Barway and Pandey (2004).

8. BVR photometry of a newly identified RS CVn binary star HD 61396 : Barway, Pandey and Padmakar (2004).

So far seven students have been awarded $\mathrm{PhD}$ degrees for their work in the different topics in astronomy and two of them completed their thesis work using small optical telescopes. Currently, six students are working towards their $\mathrm{PhD}$ degree in the field of astronomy. Several students of the department have been selected for research positions in different research institutions in India as well as outside India. In addition, students are encouraged to participate in a variety of activities to sustain their interest in astronomy. The activities include seminars and lectures by visiting scientists, student participation in summer schools, vacation student programmes organized by various research institutions in the country, student seminars in the department and the Young Astronomers Meet (YAM-India), as well as the IAU International School of Young Astronomers (ISYA).

All these activities have proved extremely useful in creating a conducive atmosphere for teaching and research in the department, as well as in helping several students of the department to take up a research career in astronomy.

With the objective of spreading the culture of astronomy using small optical telescopes in other colleges and universities in India, the A\&A research group at Raipur participates actively in workshops on observational astronomy, mostly sponsored by IUCAA. As a result, some colleges and university departments have acquired small telescopes and detectors and have started observational projects for their courses in astronomy. Other colleges and university departments have plans to acquire small telescopes in the near future. The Indian funding agencies are quite forthcoming in providing grants for the procurement of small optical telescopes in their bid to support the activities initiated by IUCAA.

\section{Looking ahead}

The future of observational astronomy using small telescopes in our University looks quite bright in view of the fact that the University administration has started the process of building a new observatory building and dome on the campus for housing the new 14" telescope. Professor Lakshman Chaturvedi, present vice-chancellor of the University, is very keen to get the work completed at the earliest opportunity. Once the permanent housing for 14" telescope is ready, it will be possible to resume our research programme on chromospherically active stars. Likewise, a new CCD spectrograph will be used to introduce projects on spectroscopic observations which were not possible earlier. The impact of our observational programme using small optical telescopes has so far been very encouraging, and we hope to continue this activity with even greater vigour in future. 


\section{Acknowledgements}

I thankfully acknowledge the support received from the University, various Indian funding agencies and IUCAA. Most importantly, I acknowledge the past and present students of the Physics Department, without whom I could not have continued the observational programme. I gratefully acknowledge a travel grant received from IAU, which made possible my participation in SPS5 and the XXVIth IAU General Assembly.

\section{References}

Barway S. 2006, Ph.D. thesis, Pt. Ravishankar Shukla University, Raipur, India

Barway, S. \& Pandey, S.K. 2004, IBVS 5553, 1, (astro-ph No. 0408351)

Barway S., Pandey S.K., Padmakar 2004, NewA. 10, 109, (astro-ph No. 0408177)

Paczynski, B. 2006, Astronomical Society of the Pacific 118, 1621

Padmakar, 2000, Ph.D. thesis, Pt. Ravishankar Shukla University, Raipur, India

Padmakar \& Pandey, S.K. 1996a, ApESSS 235, 337

Padmakar \& Pandey, S.K. 1996b, BASI 24, 717

Padmakar \& Pandey, S.K. 1999a, BASI 27, 117

Padmakar \& Pandey, S.K. 1999b, A\&AS 138, 203

Padmakar, Barway S., Pandey S.K. 2000a, BASI 28, 437

Padmakar, Singh K.P., Drake S.A., Pandey S.K. 2000b, MNRAS 314, 733

Percy, J.R. 1986, The study of variable stars using small telescopes (Cambridge University Press)

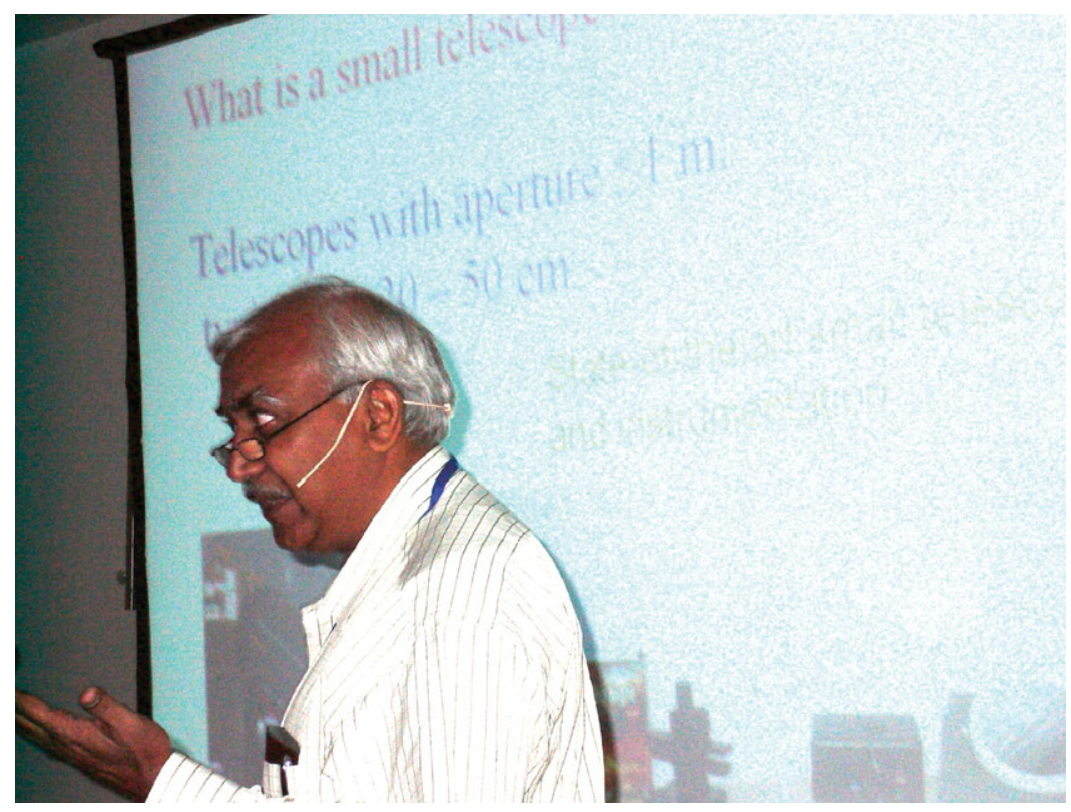

Shiva Pandey 


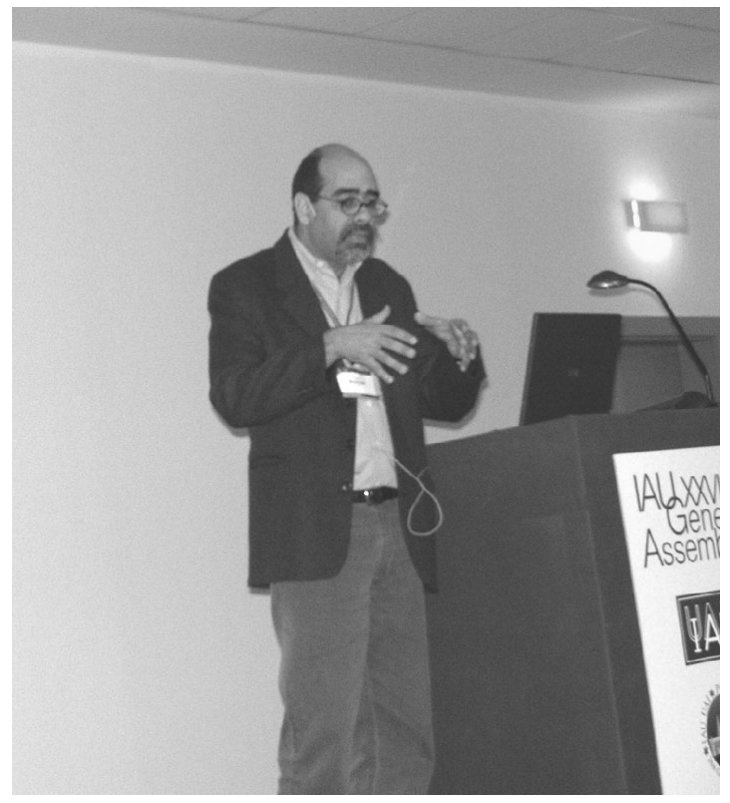

\section{Paulo Bretones}

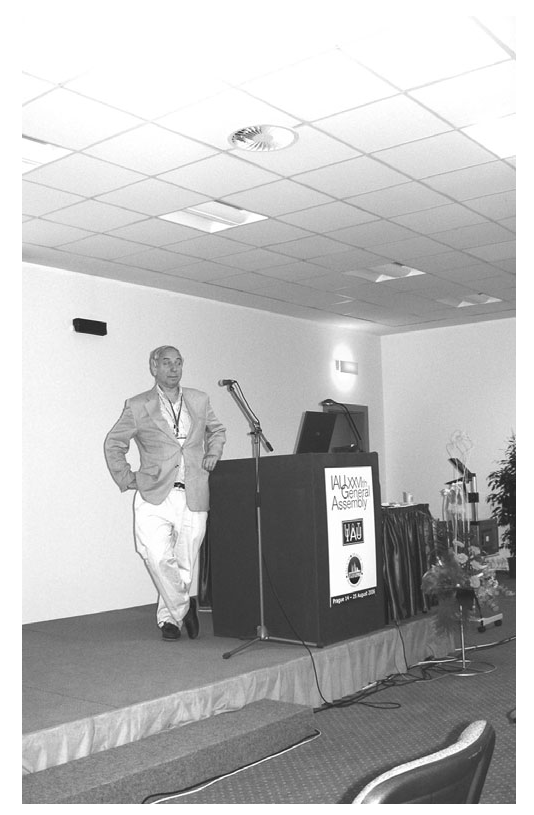

Jay Pasachoff 